\title{
Resolvents, Semigroups and Gibbs States for Infinite Coupling Constant
}

\author{
W. R. Schneider \\ Brown Boveri Research Center, Baden, Switzerland \\ Received February 20, 1975; in revised form April 14, 1975
}

\begin{abstract}
The infinite coupling constant limit of the resolvent, the semigroup and the Gibbs state is obtained for a certain class of perturbations.

As an example the infinite intrasite repulsion limit of the one-dimensional Hubbard model with nearest neighbour hopping terms is treated. This system exhibits a phase transition in the thermodynamic limit.
\end{abstract}

\section{Introduction}

In contrast to the usual perturbation theory [1] where the behaviour under a "small" perturbation is studied it is the aim of this paper to investigate the influence of "large" perturbations. To be more specific, we shall investigate the behaviour of the resolvent, the semigroup and the Gibbs state associated with $\kappa^{-1} A+B$ as $\kappa$ tends to zero. The results are contained in Theorem 1 to 3 of Section 2.

In Section 3 the results of Section 2 are applied to the one-dimensional Hubbard model (nearest neighbour hopping) with infinite intrasite repulsion. It is shown that this system may be described as a composition of a system of spinless fermions with a spin system without interaction between the two subsystems. The thermodynamic limit is calculated explicitly exhibiting a phase transition (nonanalyticity at zero chemical potential).

\section{General Theory}

Let $A$ and $B$ be operators on a Hilbert space $\mathscr{H}$ with the properties

$$
A=A^{*}, \quad B \subset B^{*}, \quad D(B) \supset D(A) .
$$

According to the closed graph theorem $B$ is $A$-bounded, i.e. there exists a constant $\alpha>0$ such that

$$
\alpha\|B u\| \leqq\|A u\|+\|u\|
$$

for all $u \in D(A)$. The operator

$$
H_{\kappa}=\kappa^{-1} A+B
$$

is self-adjoint on $D(A)$ for real $\kappa \neq 0$ with $|\kappa|<\alpha[1]$. Let $P=E(\{0\})$ where $E$ is the spectral measure associated with $A$, and $d=\operatorname{dist}(0, \operatorname{supp} E \backslash\{0\})$. As a prefix $d$ means norm $(d>0)$ or strong $(d=0)$. 
Lemma 1. For $\operatorname{Im} z \neq 0$

$d-\lim _{\kappa \rightarrow 0} B\left(z-\kappa^{-1} A\right)^{-1}=\frac{1}{z} B P$.

If $A$ and $\kappa$ are positive (4) holds for $z \notin \mathbb{R}_{+}$.

Proof. In $|\operatorname{Im} z| \geqq \varepsilon|z|(\geqq \varepsilon \operatorname{Re} z$ for $A$ positive), $0<\varepsilon<1$,

$d-\lim _{z \rightarrow 0} z(z-A)^{-1}=P$

holds. From $\left(z-\kappa^{-1} A\right)^{-1}=z^{-1}(\kappa z)(\kappa z-A)^{-1}$ it follows that (4) is valid for $B=I$. The general case is obtained from the resolvent equation

$$
B\left(z-\kappa^{-1} A\right)^{-1}=z(1-\kappa) B(z-A)^{-1}\left(z-\kappa^{-1} A\right)^{-1}+\kappa B(z-A)^{-1} .
$$

Let $\sigma\left(H_{\kappa}\right)$ be the spectrum of $H_{\kappa}$ and

$$
\Delta(z)=\lim _{\kappa \rightarrow 0} \inf \operatorname{dist}\left(z, \sigma\left(H_{\kappa}\right)\right) \text {. }
$$

Obviously, $\Delta(z)>0$ for $\operatorname{Im} z \neq 0$ and $\Delta(z)>0$ for $z \notin \mathbb{R}_{+}$if $A, B$ and $\kappa$ are positive.

Theorem 1. For $\Delta(z)>0$ the resolvent $(z-P B P)^{-1}$ exists in the subspace $P \mathscr{H}$ and

$$
d-\lim _{\kappa \rightarrow 0}\left(z-H_{\kappa}\right)^{-1}=(z-P B P)^{-1} P .
$$

Proof. Let $\Delta\left(z_{0}\right)>0$. Then

$$
\lim _{\kappa \rightarrow 0} \sup \left\|\left(z_{0}-H_{\kappa}\right)^{-1}\right\|=1 / \Delta\left(z_{0}\right)
$$

and the series

$$
\left(z-H_{\kappa}\right)^{-1}=\sum_{n=0}^{\infty}\left(z_{0}-z\right)^{n}\left(z_{0}-H_{\kappa}\right)^{-n-1}
$$

converges absolutely for all $z$ with $\left|z-z_{0}\right|<\Delta\left(z_{0}\right)$ and uniformly with respect to $\kappa$ for sufficiently small $\kappa$. If (5) holds for $z=z_{0}$ this implies $\left\|\left(z_{0}-P B P\right)^{-1}\right\| \leqq 1 / \Delta\left(z_{0}\right)$, hence

$$
(z-P B P)^{-1}=\sum_{n=0}^{\infty}\left(z_{0}-z\right)^{n}\left(z_{0}-P B P\right)^{-n-1}
$$

for $\left|z-z_{0}\right|<\Delta\left(z_{0}\right)$. As limit and sum in (6) are interchangeable (5) holds for all $z$ with $\left|z-z_{0}\right|<\Delta\left(z_{0}\right)$. It remains to prove (5) for two points in the upper and lower half-plane, respectively.

For purely imaginary $z$ it follows from (2) that

$$
\left\|B\left(z-\kappa^{-1} A\right)^{-1}\right\| \leqq(|\kappa|+1 /|z|) / \alpha<1
$$

for $|z|>1 / \alpha$ and $|\kappa|$ sufficiently small. According to Lemma 1 this implies $\left\|z^{-1} B P\right\|<1$ and hence

$$
\begin{aligned}
d-\lim _{\kappa \rightarrow 0}\left(z-H_{\kappa}\right)^{-1} & =d-\lim _{\kappa \rightarrow 0} \sum_{n=0}^{\infty}\left(z-\kappa^{-1} A\right)\left\{B\left(z-\kappa^{-1} A\right)^{-1}\right\}^{n} \\
& =\sum_{n=0}^{\infty} z^{-1} P\left(z^{-1} B P\right)^{n}=(z-P B P)^{-1} P .
\end{aligned}
$$

Remark. For $d>0$ a stronger result may be obtained (see Appendix). 
Theorem 2. Let $A$ and $B$ be positive. Then for $t>0$

$d-\lim _{\kappa \downarrow 0} e^{-t H_{\kappa}}=e^{-t P B P} P$.

Proof. Apply Theorem 1 to

$$
e^{-t H_{\kappa}}=\frac{1}{2 \pi i} \int_{\Gamma} e^{-t z}\left(z-H_{\kappa}\right)^{-1} d z
$$

where the path $\Gamma$ runs counterclockwise around $\mathbb{R}_{+}$.

Remark. Theorem 2 has a trivial generalization to the case where $B$ is bounded below (shift $H_{\kappa}$ and $B$ by the lower bound of $B$ ).

From now on we assume $\mathscr{H}$ to be separable.

Theorem 3. Let $A$ be positive and $B$ bounded below. If $H_{\kappa}, 0<\kappa \leqq \lambda \leqq \alpha$, has pure point spectrum and $\exp \left(-\beta H_{\lambda}\right)$ belongs to the trace class for some $\beta>0$, then $\exp \left(-\beta H_{\kappa}\right)$ and $P \exp (-\beta P B P)$ belong to the trace class and

$$
\lim _{\kappa \downarrow 0} \operatorname{tr} e^{-\beta H} \kappa=\operatorname{tr} P e^{-\beta P B P} .
$$

Proof. Let $X$ and $Y$ be self-adjoint operators with pure point spectra. If $f$ is a convex function then

$$
\left(u_{n},\left\{f(X)-f(Y)-(X-Y) f^{\prime}(Y)\right\} u_{n}\right) \geqq 0
$$

where $\left\{u_{n}\right\}$ is an orthonormal base of eigenvectors of $X$ or $Y$ [2]. Applying (9) to $X=H_{\kappa}, Y=H_{\mu}$ and $f(t)=\exp (-\beta t)$ yields

$$
\left(u_{n}, f(X) u_{n}\right) \geqq\left(u_{n}, f(Y) u_{n}\right)+\beta\left(\mu^{-1}-\kappa^{-1}\right)\left(u_{n}, A f(Y) u_{n}\right) .
$$

We choose now $\left\{u_{n}\right\}$ to be an orthonormal base of eigenvectors of $Y$. Hence, we have

$$
\left(u_{n}, A f(Y) u_{n}\right)=e^{-\beta \eta_{n}}\left(u_{n}, A u_{n}\right) .
$$

Accordingly, we get from (10)

$$
\left(u_{n}, f(Y) u_{n}\right) \leqq\left(u_{n}, f(X) u_{n}\right)
$$

for $\mu<\kappa$, i.e. with $f(X)$ also $f(Y)$ is in the trace class. Together with (7) the statements of the theorem follow.

Remark. Under the hypotheses of Theorem 3

$$
W(\beta, \kappa)=Z(\beta, \kappa)^{-1} e^{-\beta H_{\kappa}}
$$

with $Z(\beta, \kappa)=\operatorname{tr} \exp \left(-\beta H_{\kappa}\right)$ is a state (Gibbs state of inverse temperature $\beta$ associated with $H_{\kappa}$ ). If $P \neq 0$ then also

$$
W(\beta)=Z(\beta)^{-1} P e^{-\beta P B P}
$$

with $Z(\beta)=\operatorname{tr} P \exp (-\beta P B P)$ is a state satisfying

$$
W(\beta)=d-\lim _{\kappa \downarrow 0} W(\beta, \kappa) .
$$




\section{The Hubbard Model}

We consider the Fock representation of the CAR indexed by $M \times S$ where $M=\{1,2, \ldots, m\}, m \in \mathbb{N}$, and $S=\{1,-1\}$. Creation and annihilation operators will be denoted by $c_{i \sigma}^{*}$ and $c_{i \sigma}$, respectively. Setting $n_{i \sigma}=c_{i \sigma}^{*} c_{i \sigma}$ we introduce

$$
N=\sum_{M \times S} n_{i \sigma}=\sum_{k=0}^{2 m} k P_{k}
$$

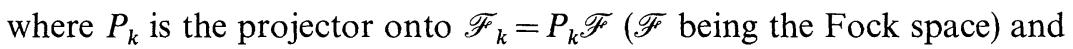

$$
Q=\sum_{i=1}^{m} n_{i, 1} n_{i,-1}=\sum_{k=0}^{2 m} \sum_{j=j_{-}(k)}^{j_{+}(k)} j P_{k, j}
$$

with $j_{-}(k)=\max \{0, k-m\}, j_{+}(k)=[k / 2]$.

The eigenspace associated with the eigenvalue $j$ of $Q P_{k}$ will be denoted by $\mathscr{F}_{k, j}=P_{k, j} \mathscr{F}$. Its dimension is given by

$$
\operatorname{dim} \mathscr{F}_{k, j}=\left(\begin{array}{c}
m \\
j
\end{array}\right)\left(\begin{array}{l}
m-j \\
k-2 j
\end{array}\right) 2^{k-2 j} .
$$

We now define the Hubbard Hamiltonian [3] by

$$
H=H_{h}+H_{m}+H_{r} .
$$

The hopping term $H_{h}$ is given by

$$
H_{h}=\sum_{(i, j) \in X} \sum_{\sigma \in S} t_{i j} c_{i \sigma}^{*} c_{j \sigma}
$$

with $X=X_{s}=\{(i, j)|| i-j \mid=1\}$ or $X=X_{r}=X_{s} \cup\{(1, m),(m, 1)\}$.

The two cases are referred to as "segment" and "ring", respectively (the model under consideration may be visualized as a system of spin $1 / 2$ fermions on a onedimensional lattice with $m$ sites forming a segment or a ring). The matrix $\left(t_{i j}\right)$ is assumed to be real symmetric.

The magnetic term $H_{m}$ is given by

$$
H_{m}=-h \sum_{i=1}^{m}\left(n_{i, 1}-n_{i,-1}\right)
$$

where $h$ is an external magnetic field.

Finally, the intrasite repulsion term is given by

$$
H_{r}=\kappa^{-1}(Q-f(N-m))
$$

with $f(x)=(x+|x|) / 2$.

The thermodynamic properties of the system are derivable from the grand partition function

$$
\Xi_{m}(\beta, \mu, h, \kappa)=\operatorname{tr} \exp (-\beta(H-\mu))=\sum_{k=0}^{2 m} e^{\beta \mu k} Z_{m, k}(\beta, h, \kappa)
$$

with

$$
Z_{m, k}(\beta, h, \kappa)=\operatorname{tr} \exp \left(-\beta P_{k} H P_{k}\right)
$$

( $\beta$ is the inverse temperature, $\mu$ the chemical potential).

Denoting limits by omitting $\kappa$ we obtain from Theorems 2 and 3 and Eqs. (18), (19) the infinite intrasite repulsion limit

$$
\Xi_{m}(\beta, \mu, h)=\sum_{k=0}^{2 m} e^{\beta \mu k} Z_{m, k}(\beta, h)
$$


and

$$
Z_{m, k}(\beta, h)=\operatorname{tr} P_{k}^{-} \exp \left\{-\beta\left(P_{k}^{-}\left(H_{h}+H_{m}\right) P_{k}^{-}\right\}\right.
$$

with $P_{k}^{-}=P_{k, j_{-}(k)}$.

To give a "physical" interpretation to this result we shall introduce a substitute system. This substitution will at the same time permit the actual computation of (20), (21).

Let $\mathscr{F}^{0}$ be the Fock space of the CAR representation indexed by $M$ with creation and annihilation operators $d_{i}^{*}$ and $d_{i}$, respectively. $\mathscr{F}_{k}^{0}=P_{k}^{0} \mathscr{F}(k=0, \ldots, m)$ denotes the $k$-particle subspace.

As Hamiltonian of the system we shall take

$$
H^{0}=\sum_{(i, j) \in X} t_{i j} d_{i}^{*} d_{j} .
$$

Furthermore, we introduce $\mathscr{H}_{n}=\mathscr{H}_{1} \otimes \ldots \otimes \mathscr{H}_{1}$, the $n$-fold tensor product of a twodimensional Hilbert space $\mathscr{H}_{1}$. Let $\left\{\phi_{\sigma}, \sigma= \pm 1\right\}$ be an orthonormal base of $\mathscr{H}_{1}$ and set $s_{j}=I \otimes \ldots \otimes s \otimes \ldots \otimes I$ with $s, s \phi_{\sigma}=\sigma \phi_{\sigma}$, as $j$-th factor. As Hamiltonian in $\mathscr{H}_{n}$ we take

$$
H_{n}=-h \sum_{j=1}^{n} s_{j} .
$$

The system $\left(\mathscr{F}^{0}, H^{0}\right)$ is a system of spinless fermions whose dynamics is described by the hopping Hamiltonian $H^{0}$ whereas $\left(\mathscr{H}_{n}, H_{n}\right)$ is a system of spins interacting only with the external magnetic field $h$.

The substitution mentioned above may now be formulated by the following theorem.

Theorem 4. For $k \leqq m$ (i.e. $j_{-}(k)=0$ ) there exists a unitary operator $U_{k}: \mathscr{F}_{k, 0} \rightarrow$ $\mathscr{F}_{k}^{0} \otimes \mathscr{H}_{k}$ such that

$$
U_{k}\left(P_{k}^{-}\left(H_{h}+H_{m}\right) P_{k}^{-}\right) U_{k}^{-1}=P_{k}^{0} H^{0} P_{k}^{0} \otimes I+I \otimes H_{k}
$$

whereas for $k>m$ (i.e. $j_{-}(k)=k-m$ ) there exists a unitary operator $U_{k}: \mathscr{F}_{k, k-m} \rightarrow$ $\widetilde{F}_{k-m}^{0} \otimes \mathscr{H}_{2 m-k}$ such that

$$
U_{k}\left(P_{k}^{-}\left(H_{h}+H_{m}\right) P_{k}^{-}\right) U_{k}^{-1}=P_{k-m}^{0} H^{0} P_{k-m}^{0} \otimes I+I \otimes H_{2 m-k} .
$$

Remark. Theorem 4 says that in the infinite intrasite repulsion limit the Hubbard system may be described (in each subspace $\mathscr{F}_{k}$ separately) as a composition of a system of spinless fermions with a spin system. The Hamiltonian of the composite system [given by the r.h.s. of Eqs. (24) and (25), respectively] contains no interaction between the two subsystems, i.e. translational and spin degrees of freedom are completely decoupled.

Proof. For $k \leqq m$ define $U_{k}$ as the extension by linearity of

$$
U_{k} c_{r_{1} \sigma_{1}}^{*} \ldots c_{r_{k} \sigma_{k}}^{*} \Omega=d_{r_{1}}^{*} \ldots d_{r_{k}}^{*} \Omega^{0} \otimes \phi_{\sigma}
$$

$\left(\phi_{\sigma}=\phi_{\sigma_{1}} \otimes \phi_{\sigma_{2}} \otimes \ldots \otimes \phi_{\sigma_{k}}\right)$ with $1 \leqq r_{1}<r_{2}<\ldots<r_{k} \leqq m$ and arbitrary $\sigma_{1}, \ldots, \sigma_{k}$. Verification of (25) is left to the reader as well as the case $k>m$. 
It follows from (21), (24), and (25) that

$$
Z_{m, k}(\beta, h)=Z_{m, k}^{0}(\beta) Z_{k}(\beta, h), \quad k \leqq m
$$

and

$$
Z_{m, k}(\beta, h)=Z_{m, k-m}^{0}(\beta) Z_{2 m-k}(\beta, h), \quad k>m
$$

where

$$
Z_{m, j}^{0}(\beta)=\operatorname{tr} \exp \left(-\beta P_{j}^{0} H^{0} P_{j}^{0}\right)
$$

and

$$
Z_{n}(\beta, h)=\operatorname{tr} \exp \left(-\beta H_{n}\right) .
$$

From (23) we obtain

$$
Z_{n}(\beta, h)=(2 \cosh \beta h)^{n} .
$$

Introducing $N^{0}=\sum d_{i}^{*} d_{i}$ and

$$
Z_{m}^{0}(\beta, v)=\operatorname{tr} \exp \left(-\beta\left(H^{0}-v N^{0}\right)\right)=\sum_{j=0}^{m} e^{\beta v j} Z_{m, j}^{0}(\beta)
$$

the grand partition function for the system of spinless fermions, we obtain collecting all results

$$
\Xi_{m}(\beta, \mu, h)=e^{\beta m(\mu+\gamma)}\left\{\Xi_{m}^{0}(\beta, \mu-\gamma)+\Xi_{m}^{0}(\beta, \mu+\gamma) e^{\beta m(\mu+\gamma)}-1\right\}
$$

with $\exp (\beta \gamma)=2 \cosh \beta h$.

The Hamiltonian $H^{0}$ is easily diagonalized and (31) yields

$$
\Xi_{m}^{0}(\beta, v)=\prod_{j=1}^{m}\left(1+e^{-\beta\left(\lambda_{j}-v\right)}\right)
$$

where $\lambda_{j}(j=1, \ldots, m)$ are the eigenvalues of the matrix $\left(t_{i j}\right)$.

As we are interested in the thermodynamic limit (i.e. $m \rightarrow \infty$ ) we assume from now on translational invariance.

As $t_{i j}=t,(i, j) \in X$, this leads to

$$
\lambda_{j}=2 t \cos \frac{\pi}{m+1} j, \quad j=1, \ldots, m
$$

in the case of the segment and

$$
\lambda_{j}=2 t \cos \frac{2 \pi}{m} j, \quad j=1, \ldots, m
$$

in the case of the ring.

For the "pressure" $p^{0}(\beta, v)=\lim m^{-1} \log \Xi_{m}^{0}(\beta, v)$ we obtain from (33) and (34) or (35)

$$
p^{0}(\beta, v)=\frac{1}{\pi} \int_{0}^{\pi} d x \log \left(1+e^{-\beta(2 t \cos x-v)}\right) .
$$

The density $\varrho^{0}(\beta, v)=\beta^{-1} p^{0}{ }_{v}(\beta, v)$ is given by

$$
\varrho^{0}(\beta, v)=\frac{1}{\pi} \int_{0}^{\pi} d x\left(e^{\beta(2 t \cos x-v)}+1\right)^{-1} .
$$


Finally, the "pressure" $p(\beta, v, h)$ and the density $\varrho(\beta, \mu, h)$ for the Hubbard model in the infinite repulsion limit are obtainable from (32). This leads to

$$
p(\beta, \mu, h)=\beta(\mu+\gamma)+p^{0}(\beta,|\mu|-\gamma)
$$

and

$$
\varrho(\beta, v, h)=1+\operatorname{sign}(\mu) p^{0}(\beta,|\mu|-\gamma) .
$$

Obviously, $p$ and $\varrho$ as functions of $\mu$ are real analytic in the complement of $\mu=0$ but not at $\mu=0$. The density $\varrho$ has a jump at $\mu=0$ given by $\Delta \varrho(\beta, h)=2 \varrho^{0}(\beta,-\gamma)$.

It follows from (37) that $\Delta \varrho(0, h)=2 / 3$ and

$$
\lim _{\beta \rightarrow \infty} e^{\beta|h|} \Delta \varrho(\beta, h)=1,
$$

i.e. for $h \neq 0$ the jump vanishes as the temperature approaches zero whereas for $h=0$ it remains finite.

As $\varrho^{0}(\beta, v)$ increases monotonically from 0 to 1 as $v$ varies from $-\infty$ to $\infty$ it follows that $\varrho(\beta, \mu, h)$ increases from 0 to $1-\varrho^{0}(\beta,-\gamma)$ and from $1+\varrho^{0}(\beta,-\gamma)$ to 2 as $\mu$ varies from $-\infty$ to 0 and from 0 to $\infty$, respectively.

The magnetic properties of the system are displayed by the magnetization

$$
m(\beta, v, h)=\frac{1}{\beta} \frac{\partial}{\partial h} p(\beta, \mu, h)=(1-\varrho(\beta, \mu, h)-1 \mid) \tanh \beta h
$$

and the susceptibility

$$
\chi(\beta, \mu)=\frac{\partial}{\partial h} m(\beta, \mu, h)_{h=0}=(1-\lfloor\varrho(\beta, \mu, 0)-1 \mid) \beta,
$$

i.e. the typical behaviour of a system of noninteracting spins in an external field.

Remark. The infinite intrasite repulsion limit has been treated before by several authors [4-6]. Their results are in qualitative agreement with ours, the difference lies in the rigour of the mathematical methods used to obtain them.

Acknowledgements. I would like to thank Sigfrid Strässler for drawing my attention to this subject, Jacob Bernasconi and Hans J. Wiesmann for helpful conversations, Sergio Albeverio and Raphael Höegh-Krohn for encouraging remarks and (last but not least) Walter Hunziker for the version of Theorem 1 given here.

\section{Appendix}

We shall prove a theorem which essentially says that $\left(z-H_{\kappa}\right)^{-1}$ is a real analytic function of $\kappa$ in a neighbourhood of $\kappa=0$ if $d>0$ (notation and assumptions as in Section 2).

Theorem 5. Let $d>0$. Then to each $z \in \varrho^{\prime}(P B P)$ (the resolvent set of $P B P$ considered as operator in $P \mathscr{H})$ there exists $\kappa_{1}>0$ such that the relation

$$
\left(z-H_{\kappa}\right)^{-1}=R(z)+\kappa E(z) F(\kappa, z) G(z)
$$

holds for $-\kappa_{1}<\kappa<\kappa_{1}$ where

$$
F(\kappa, z)=\sum_{n=0}^{\infty}(\kappa F(z))^{n}
$$


is absolutely convergent and $R(z), E(z), F(z), G(z)$ are given by

$$
\begin{aligned}
& R(z)=(z-P B P)^{-1} P, \\
& E(z)=S+R(z) B S, \\
& F(z)=(z-B) E(z), \\
& G(z)=(z-B) R(z)-I,
\end{aligned}
$$

with

$$
S=n-\lim _{z \rightarrow 0}(z-A)^{-1}(P-I) .
$$

Proof. As $d>0$ (A7) makes sense and the norm of $S$ satisfies $d\|S\|=1$. Furthermore, we have $S \mathscr{H} \subset D(A)$. This is seen as follows: Let $f \in \mathscr{H}$ and $g(z)=$ $(z-A)^{-1}(P-I) f$. By definition we have

$$
\lim _{z \rightarrow 0} g(z)=S f
$$

and from $A g(z)=(I-P) f+z g(z)$ it follows that

$$
\lim _{z \rightarrow 0} A g(z)=(I-P) f \text {. }
$$

As $A$ is closed Eqs. (A8) and (A9) imply $S f \in D(A)$ and $A S f=(I-P) f$, i.e.

$$
A S=I-P \text {. }
$$

The counterpart to (A10) is

$$
S A=(I-P) \mid D(A)
$$

which follows from

$$
(z-A)^{-1}(P-I) A=\left\{I-z(z-A)^{-1}\right\} \mid D(A)
$$

and from $n-\lim _{z \rightarrow 0} z(z-A)^{-1}=P$.

By the closed graph theorem $B S$ and $B P$ are bounded. Hence, by (A4)-(A6), also $E(z), F(z)$ and $G(z)$ are bounded.

The series (A2) is absolutely convergent for $|\kappa|<\kappa_{2}$ where $\kappa_{2}\|F(z)\|=1$. Using (A10), (A11) and $P S=P F(z)=P G(z)=0$ we obtain

$$
\left(z-H_{\kappa}\right)\{R(z)+\kappa E(z) F(\kappa, z) G(z)\}=I
$$

and

$$
\{R(z)+\kappa E(z) F(\kappa, z) G(z)\}\left(z-H_{\kappa}\right)=I \mid D(A) .
$$

For $-\kappa_{1}<\kappa<\kappa_{1}$ with $\kappa_{1}=\min \left(\alpha, \kappa_{2}\right)$ (A1) follows from (A12) and (A13).

Remark 1. For $B=0$ (A1) reduces to

$$
\left(z-\kappa^{-1} A\right)^{-1}=\frac{P}{z}-\kappa S \sum_{n=0}^{\infty}(\kappa z S)^{n} .
$$

As $\varrho^{\prime}(P B P)=\mathbb{C} \backslash\{0\}$ Theorem 5 says that to $z \neq 0$ there exists $\kappa_{1}>0$ such that (A14) holds for $-\kappa_{1}<\kappa<\kappa_{1}$ (we may choose $\kappa_{1}\|z S\|=1$ ). For $\kappa=1$ and $\|z S\|<1$ (A14) is identical to the standard Laurent expansion of $(z-A)^{-1}$. 
Remark 2. If 0 is in the resolvent set of $A$ then $P=0$ and (A1) reduces to

$$
\left(z-H_{\kappa}\right)^{-1}=-\kappa A^{-1} \sum_{n=0}^{\infty}\left(\kappa(z-B) A^{-1}\right)^{n}
$$

which is the Neumann series of

$$
\left(z-H_{\kappa}\right)^{-1}=-\left\{\left(I-\kappa(z-B) A^{-1}\right) \kappa^{-1} A\right\}^{-1} .
$$

As $P=0$ we have $\varrho^{\prime}(P B P)=\mathbb{C}$, i.e. to $z \in \mathbb{C}$ there exists $\kappa_{1}>0$ such that (A15) holds for $-\kappa_{1}<\kappa<\kappa_{1}$.

\section{References}

1. Kato, T.: Perturbation theory for linear operators. Berlin-Heidelberg-New York: Springer 1966

2. Ruelle, D.: Statistical mechanics. New York: Benjamin 1969

3. Hubbard, J.: Proc. Roy. Soc. A 276, 238-257 (1963); A 277, 237-259 (1964)

4. Beni, G., Holstein, T., Pincus, P.: Phys. Rev. B 8, 312-316 (1973)

5. Klein, D. J.: Phys. Rev. B 8, 3452-3458 (1973)

6. Sokoloff, J. B.: Phys. Rev. B 2, 779-781 (1970)

Communicated by W. Hunziker

W. R. Schneider

Brown Boveri Research Center

CH-5401 Baden, Switzerland 
